\title{
Implementation of Bus Bar Switching and Short Circuit Constraints in Optimal Power Flow Problems
}

\author{
T. Van Acker \\ D. Van Hertem \\ Research Division ELECTA \\ Department of Electrical Engineering (ESAT) \\ KU Leuven \\ Leuven, Belgium \\ tom.vanacker@esat.kuleuven.be
}

\author{
D. Bekaert \\ K. Karoui \\ C. Merckx \\ Power System Consulting \\ Tractebel Engineering (GDF Suez) \\ Sint-Lambrechts-Woluwe, Belgium \\ david.bekaert@gdfsuez.com
}

\begin{abstract}
The topology of an electric network heavily influences power system operations, power flows, short circuit currents and certain reliability aspects. While meshing increases reliability, it also increases the short circuit power, which can be a problem especially in highly meshed grids. Bus bar switching is often employed by the system operator for preventive and corrective actions, as it is a quasi-free control action. The introduction of binary variables, representative of the state of the circuit breakers, in the optimization description allows for bus bar switching resulting in the adaptation of the topology of a grid. The goal of this paper is to present an algorithm that is able to propose a topology that complies with the Short Circuit Constraints (SCC) whilst respecting the Power Flow Constraints (PFC). Both are adapted in such a way that they allow binary switching actions. The mathematical description of the optimization problem is a Mixed Integer Non Convex Quadratic Constraint Program (MINCQCP) and is implemented in AMPL using the Couenne solver. The paper concludes with two tests that show the functionality of the approach.
\end{abstract}

Index Terms-MINCQCP, OPF Problems, Short Circuit Calculations, Transmission Switching

\section{NOMENCLATURE}

\section{A. Abbreviations}

$\begin{array}{ll}\text { CB } & \text { Circuit Breaker } \\ \text { DC OPF } & \begin{array}{l}\text { DC Optimal Power Flow } \\ \text { Mixed Integer Non Convex Quadratic } \\ \text { Constraint Problem }\end{array} \\ \text { NC } & \text { Node Constraints } \\ \text { NSEC } & \text { Non-Switchable Elements Constraints } \\ \text { PFC } & \text { Power Flow Constraints } \\ \text { SCC } & \text { Short Circuit Constraints } \\ \text { SEC } & \text { Switchable Elements Constraints }\end{array}$

The ELECTA research group of KU Leuven is a founding member of the EnergyVille research lab

\section{B. Variables}

I

$\mathrm{N}_{S}$

$\mathrm{N}_{B}$

$\mathrm{N}_{G}$

$\mathrm{P}$

U

Y

$\mathrm{Y}_{b, i j}$

$\mathrm{Y}_{c}$

$\delta$

$\delta_{i j}$

$\theta$

C. Sets

b

c

e

g

1

$\mathrm{n}$

sc

CS

\author{
Current vector \\ Number of bus bar nodes \\ Number of branch nodes \\ Number of generator nodes \\ Active power \\ Voltage vector \\ Admittance matrix \\ Admittance of a branch with nodes i \& j \\ Admittance of a circuit breaker \\ Binary state variable of a $\mathrm{CB}$ \\ $\delta$ of a $\mathrm{CB}$ with nodes $\mathrm{i} \& \mathrm{j}$ \\ Voltage angle \\ Set of branches \\ Set of circuit breakers \\ Set of elements $=\{b, g, l\}$ \\ Set of generators \\ Set of loads \\ Set of nodes \\ Set of short circuits \\ Set of considered substations
}

\section{INTRODUCTION}

The increased penetration of renewable energy sources over the last decade has resulted in the need for more transmission capacity, leading to more complex and dense power systems. Besides the advantage of more capacity, the expansion of a power system has also some disadvantages [1]. One of the main disadvantages is the increase of the short circuit level, sometimes beyond the capabilities of the existing circuit breakers.

Several possibilities [2] exist to limit the short circuit level:

- Current limiting equipment (e.g. current limiting reactors)

- Replacing existing circuit breakers

- Corrective switching (e.g. bus bar switching) 
Both the implementation of current limiting equipment [3] and replacing the existing circuit breakers come at a considerable cost.

Another option, bus bar switching, is a quasi-free option to reduce the short circuit level [4]-[6]. However, it could be the case that a switching operation in order to reduce the short circuit level at one substation induces a higher short circuit level in an other substation. In order to avoid this, the impact of switching actions on other substations should be taken into account [7].

This paper presents an algorithm that allows bus bar switching actions and is able to optimize the topology of any meshed electricity grid taking into account both Power Flow Constraints (PFC) and Short Circuit Constraints (SCC). The basis for the proposed algorithm is a DC Optimal Power Flow (DC OPF). A DC OPF is a linearized version of the AC OPF [8]. Bus bar switching, as it is used in this paper, includes two possible switching actions. The first entails that an element (e.g. generator, branch or load) is disconnected from one bus bar of a substation and reconnected to another bus bar of the same substation. The second switching action connects or disconnects the bus bars of a substation. Bus bar switching is made possible by the introduction of binary variables in the optimization description that represent the state of the circuit breakers, allowing for decisions with respect to the grid topology. The PFC are the typical constraints added in an OPF problem, ensuring that Kirchhoff's laws and the physical limitations of the grid elements are respected. The SCC ensure that the short circuit power at the nodes stays within a specific range guaranteeing that the circuit breakers are able to interrupt any short circuit. A proof of concept for the algorithm is given by the implementation in Matlab [9]. Reducing the short circuit power at a node generally entails opening certain circuit breakers. This reduces the reliability of the grid and could cause islanding in the system in case of subsequent contingencies. In order to avoid or minimize this, several measures are introduced in this paper. A first measure is to maximize the number of closed breakers in order to maximize the reliability of the system. Another measure is to test the solutions for the occurrence of islanding. The developed algorithm is a Mixed Integer Non Convex Quadratic Constraint Program, these problems are in NP-complete [10]. The computational time of those problems increases at most exponentially with the size. A heuristic scheme is introduced which significantly reduces the computational time required to find the an optimal topology. The developed algorithm is implemented in AMPL using Couenne as a solver. AMPL is an algebraic modeling language for describing and solving high-complexity problems for large-scale mathematical computation [11]. Couenne is a solver that aims at finding global optima of non-convex MINLPs. It implements linearization, bound reduction and branching methods within a branch-andbound framework [12].

The paper is organized as follows. In section II, the possibility of switching operations is introduced using binary decision variables. Section III discusses the influence of the introduc- tion of those binary decision variables on the PFC. Section IV presents a way to determine the SCC. In section $\mathrm{V}$, the mathematical description of the entire problem is presented. Section VI describes the usage of the developed algorithm on an industrial substation with ten bus bars. In section VII, the algorithm is applied to a power system with fourteen substations, each with two bus bars. Section VIII concludes this paper.

\section{BUS BAR SWITCHING}

In a standard DC OPF, a substation is often considered to be a single node. When bus bar switching is considered, this is no longer the case.

A substation consists of one or multiple bus bars. All incoming and outgoing branches, generators and loads are connected to one or all bus bars of a substation with a separate circuit breaker (see Fig. 1). The bus bars of a substation can also be connected to each other by a circuit breaker.

In order to make bus bar switching possible, a binary variable $\delta$ is introduced for each breaker, representative of the state of that circuit breaker. A closed breaker is represented by a $\delta$ that is equal to one, an open breaker by a $\delta$ equal to zero.

For the purpose of this paper, a substation consists of nodes (including bus bar nodes) and two types of elements: switchable elements (i.e. circuit breakers) and non-switchable elements (i.e. generators, branches and loads). The distinction between the two types of elements is made because the PFC for both elements are different due to the possible presence of the binary variable $\delta$.

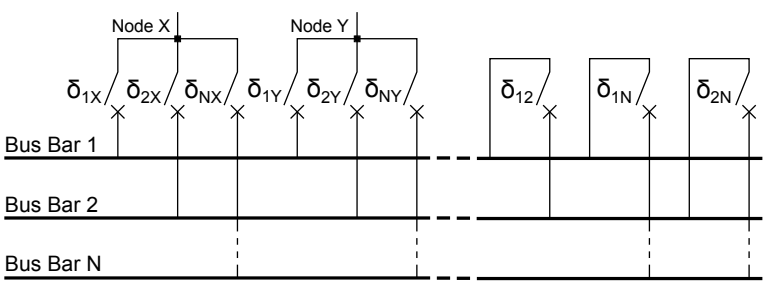

Figure 1. Switchgear arrangement in a substation with $\mathrm{N}$ bus bars

\section{POWER Flow CONSTRAINTS}

The PFC can be divided into three distinct groups: Node Constraints (NC), Non-Switchable Element Constraints (NSEC) and Switchable Element Constraints (SEC).

\section{A. Node Constraints}

The NC consists of two types of constraints. The first type of NC makes sure that the voltage angle of all nodes $\mathrm{n}$ remains within limits (1). The second type of NC guarantees that the first Kirchhoff law is respected in all nodes $n(2)$.

$$
\begin{array}{ll}
\theta_{n}^{\text {min }} \leq \theta_{n} \leq \theta_{n}^{\max } & \forall \mathrm{n} \\
\Sigma_{b} P_{b, n}+\Sigma_{g} P_{g, n}+\Sigma_{l} P_{l, n}+\Sigma_{c} P_{c, n}=0 & \forall \mathrm{n}
\end{array}
$$




\section{B. Non-Switchable Element Constraints}

Branches and generators are the non-switchable elements considered for the NSEC. The NSEC ensure that the generator active power (3) and the branch flows (4) remain within limits. Equation (5) defines the active power flows for all branches $b$ with an admittance $Y_{b}$.

$$
\begin{array}{ll}
P_{g}^{\text {min }} \leq P_{g} \leq P_{g}^{\text {max }} & \forall \mathrm{g} \\
P_{b}^{\text {min }} \leq P_{b} \leq P_{b}^{\text {max }} & \forall \mathrm{b} \\
Y_{b}\left(\theta_{\text {in }}-\theta_{\text {out }}\right)-P_{b}=0 & \forall \mathrm{b}
\end{array}
$$

\section{Switchable Element Constraints}

Every circuit breaker is considered to be a switchable element. The SEC are based on the constraints of non-switchable branches but are adapted to allow for the closing or opening of the circuit breakers based on their $\delta$. The first type of SEC ensures that the active power through all circuit breakers $\mathrm{c}$ remains within the allowed limits when it is closed $(\delta=1)$. When the circuit breaker is open $(\delta=0)$, this constraint sets the active power through this breaker to zero (6). The second type of SEC defines the active power flow for all circuit breakers c when the breaker is closed (7). The admittance of a circuit breaker $Y_{c}$ is significantly larger than that of a branch. For the purpose of this paper, $Y_{c}$ is set to $10^{6} \mathrm{~S}$ for all circuit breakers.

$$
\begin{array}{ll}
\delta P_{c}^{\text {min }} \leq P_{c} \leq \delta P_{c}^{\max } & \forall \mathrm{c} \\
\delta Y_{c}\left(\theta_{\text {in }}-\theta_{\text {out }}\right)-P_{c}=0 & \forall \mathrm{c}
\end{array}
$$

In contrast to the linear character of the NC and NSEC, (7) are mixed integer non convex quadratic constraints due to the presence of the term $\delta\left(\theta_{\text {in }}-\theta_{\text {out }}\right)$.

\section{SHORT Circuit CONSTRAINTS}

The SCC are constructed using the superposition method [13]. The superposition method is based on Ohm's law:

$$
\mathbf{I}=Y \mathbf{U}
$$

For each possible short circuit (i.e. for each bus bar node), this matrix equation is constructed. The admittance matrix $\mathrm{Y}$ remains unchanged while the voltage and current vector change for each possible short circuit. In order to construct the Y-matrix, U- and I-vector, the considered nodes are classified into three groups: bus bar nodes $\mathrm{S}$, branch nodes $\mathrm{B}$ and generator nodes $G$. In this section, the construction of these elements is discussed and as a last part, this method is demonstrated with a small example.

\section{A. Admittance Matrix $Y$}

Due to the distinction between the different types of nodes, the Y-matrix can be divided into 9 submatrices.

$$
Y=\left[\begin{array}{ccc}
Y_{S S} & Y_{S B} & Y_{S G} \\
Y_{S B}^{T} & Y_{B B} & Y_{B G} \\
Y_{S G}^{T} & Y_{B G}^{T} & Y_{G G}
\end{array}\right]
$$

The size, content and formulation of the submatrices is described in Table I.
Table I

Submatrices OF ADMITTANCE MATRIX Y

\begin{tabular}{llll} 
Name & Size & Content & Formulation \\
\hline$Y_{S S}$ & $N_{S} \times N_{S}$ & CB between bus bars & $\delta_{i j} Y_{c}$ \\
$Y_{B B}$ & $N_{B} \times N_{B}$ & All branches of the network & $Y_{b, i j}$ \\
$Y_{G G}$ & $N_{G} \times N_{G}$ & All zero matrix & \\
$Y_{S B}$ & $N_{S} \times N_{B}$ & CB between branches and bus bars & $\delta_{i j} Y_{c}$ \\
$Y_{S G}$ & $N_{S} \times N_{G}$ & CB between generators and bus bars & $\delta_{i j} Y_{c}$ \\
$Y_{B G}$ & $N_{B} \times N_{G}$ & All zero matrix & \\
\hline
\end{tabular}

The diagonal elements of the Y-matrix are the negative summation of all the elements connected to the respective diagonal element.

$$
Y_{i i}=-\sum_{j=1, i \neq j}^{N_{s}+N_{b}+N_{g}} Y_{i j}
$$

\section{B. Voltage Vector $\boldsymbol{U}$}

$\mathbf{U}$ is composed of three subvectors: $\mathbf{U}_{S}, \mathbf{U}_{B}$ and $\mathbf{U}_{G} \cdot \mathbf{U}_{S}$ consists of the voltage of the short circuited node $\mathrm{U}_{S C}$ and the other bus bar voltages $\mathbf{U}_{S^{\prime}}(*)$. The position of the voltage of the short circuited node depends on the identification number of that node, $U_{S C}$ is located at the first position for the first node, second position for the second node and so on. The voltage of the short circuited node is set to 0 p.u., the generator voltages to 1 p.u. and all other voltages are unknown variables $(* *)$.

$$
\mathbf{U}=\left[\begin{array}{c}
\mathbf{U}_{S} \\
\mathbf{U}_{B} \\
\mathbf{U}_{G}
\end{array}\right] \stackrel{*}{=}\left[\begin{array}{c}
U_{S C} \\
\mathbf{U}_{S^{\prime}} \\
\mathbf{U}_{B} \\
\mathbf{U}_{G}
\end{array}\right] \stackrel{* *}{=}\left[\begin{array}{c}
0 \\
\mathbf{U}_{S^{\natural}} \\
\mathbf{U}_{B} \\
\mathbf{1}
\end{array}\right]
$$

An additional constraint (12) is introduced to keep the voltages at each node $\mathrm{n}$ between 0 and 1 p.u. for all short circuits sc, in order to reduce the search space.

$$
0 \leq U_{n} \leq 1 \quad \forall \mathrm{sc} \& \forall \mathrm{n}
$$

\section{Current Vector I}

$\mathbf{I}$ is composed of three subvectors: $\mathbf{I}_{S}, \mathbf{I}_{B}$ and $\mathbf{I}_{G}$. $\mathbf{I}_{S}$ consists of the short circuit current at the short circuited node $\mathrm{I}_{S C}$ and the currents injected at the other bus bars $\mathbf{I}_{S^{\prime}}(*)$. The position of the short circuit current follows the same logic as the short circuit voltage in the $\mathbf{U}$-vector. The short circuit current is an unknown variable, the same goes for the current injected at the generator nodes. At all other nodes, no current is injected or extracted, these currents can be set to 0 p.u. (**).

$$
\mathbf{I}=\left[\begin{array}{c}
\mathbf{I}_{S} \\
\mathbf{I}_{B} \\
\mathbf{I}_{G}
\end{array}\right] \stackrel{*}{=}\left[\begin{array}{c}
I_{S C} \\
\mathbf{I}_{S^{\prime}} \\
\mathbf{I}_{B} \\
\mathbf{I}_{G}
\end{array}\right] \stackrel{* *}{=}\left[\begin{array}{c}
I_{S C} \\
0 \\
0 \\
\mathbf{I}_{G}
\end{array}\right]
$$

In order to keep the short circuit currents limited to a value which is switchable by the circuit breakers, an additional 
constraint (14) is introduced for each possible short circuit sc.

$$
I_{S C}^{\min } \leq I_{S C} \leq I_{S C}^{\max } \quad \forall \mathrm{sc}
$$

\section{SCC of a Small Grid}

In order to illustrate the construction of the SCC, a test grid is introduced in Fig. 2 consisting of two substations with each two bus bars, one branch and one generator.

A short circuit is applied to the second bus bar of the second substation (i.e. node 4), meaning that $\mathrm{I}_{S C}$ and $\mathrm{U}_{S C}$ are shifted to the fourth position of their respective vectors.

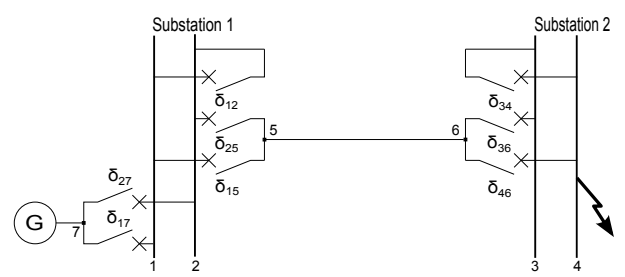

Figure 2. Test grid used for demonstrating the SCC

The following matrix equation describes the short circuit on the second bus bar of the second substation:

$$
\left[\begin{array}{c}
0 \\
0 \\
0 \\
I_{S C} \\
0 \\
0 \\
I_{G}
\end{array}\right]=\left[\begin{array}{cccc|cc|c}
-\Sigma & \delta_{12} Y_{c} & 0 & 0 & \delta_{15} Y_{c} & 0 & \delta_{17} Y_{c} \\
\delta_{12} Y_{c} & -\Sigma & 0 & 0 & \delta_{25} Y_{c} & 0 & \delta_{27} Y_{c} \\
0 & 0 & -\Sigma & \delta_{34} Y_{c} & 0 & \delta_{36} Y_{c} & 0 \\
0 & 0 & \delta_{34} Y_{c} & -\Sigma & 0 & \delta_{46} Y_{c} & 0 \\
\hline \delta_{15} Y_{c} & \delta_{25} Y_{c} & 0 & 0 & -\Sigma & Y_{b, 56} & 0 \\
0 & 0 & \delta_{36} Y_{c} & \delta_{46} Y_{c} & Y_{b, 56} & -\Sigma & 0 \\
\hline \delta_{17} Y_{c} & \delta_{27} Y_{c} & 0 & 0 & 0 & 0 & -\Sigma
\end{array}\right]\left[\begin{array}{c}
U_{1} \\
U_{2} \\
U_{3} \\
0 \\
U_{5} \\
U_{6} \\
1
\end{array}\right]
$$

The constraints generated using the superposition method, as described in this section, are mixed integer non-convex quadratic constraints, where the quadratic elements are of the form $\delta * U$.

\section{Formulation of the Entire Problem}

In this section, the formulation of the entire problem is presented. This formulation allows topology changes and contains both the PFC and SCC.

$$
\text { minimize objective function }
$$

subject to

$\begin{array}{lll}\text { NC } & \theta_{n}^{\text {min }} \leq \theta_{n} \leq \theta_{n}^{\text {max }} & \forall \mathrm{n} \\ & \Sigma_{b} P_{b}+\Sigma_{g} P_{g}+\Sigma_{l} P_{l}+\Sigma_{c} P_{c}=0 & \forall \mathrm{n} \\ \text { NSEC } & P_{g}^{\text {min }} \leq P_{g} \leq P_{g}^{\text {max }} & \forall \mathrm{g} \\ & P_{b}^{\text {min }} \leq P_{b} \leq P_{b}^{\text {max }} & \forall \mathrm{b} \\ & Y_{b}\left(\theta_{\text {in }}-\theta_{\text {out }}\right)-P_{b}=0 & \forall \mathrm{b} \\ \text { SEC } & \delta P_{c}^{\text {min }} \leq P_{c} \leq \delta P_{c}^{\text {max }} & \forall \mathrm{c} \\ & \delta Y_{c}\left(\theta_{\text {in }}-\theta_{\text {out }}\right)-P_{c}=0 & \forall \mathrm{c} \\ \text { SCC } & \mathbf{I}_{s c}=Y \mathbf{U}_{s c} & \forall \mathrm{sc} \\ & 0 \leq U_{n} \leq 1 & \forall \mathrm{sc} \& \forall \mathrm{n} \\ & I_{S C}^{\text {min }} \leq I_{S C} \leq I_{S C}^{\max } & \forall \mathrm{sc}\end{array}$

This optimization problem is an Mixed Integer Non Convex Quadratic Constraint Problem (MINCQCP). The problem is implemented in AMPL using the Couenne solver.

\section{Test Case I: Industrial Substation}

In this section, a first application for the developed algorithm is discussed. An industrial substation with two short circuit limiting reactors is considered. The purpose of this test is to see if the developed algorithm is able to find an alternative topology for this substation using bus bar switching actions. In order to ensure maximal possible reliability and nonoccurrence of islanding, some additions to the algorithm are presented.

\section{A. Industrial Substation}

The substation, depicted in Fig. 3, consists of ten bus bars, fourteen generators and four large loads. The bus bars of this substation experience a too high short circuit power (330 p.u.). To limit the short circuit power, two short circuit limiting reactors $\mathrm{C} 1$ and $\mathrm{C} 2$ were installed, respectively between bus bars S1C and S1D and between bus bars S1H and S1I, reducing the short circuit power to 242 p.u.. The substation is connected to bus bar S2A through two branches B1 and B2. This bus bar is then further connected to what can be described as an infinite grid with a short circuit power $S_{S C}$ equal to 190 p.u..

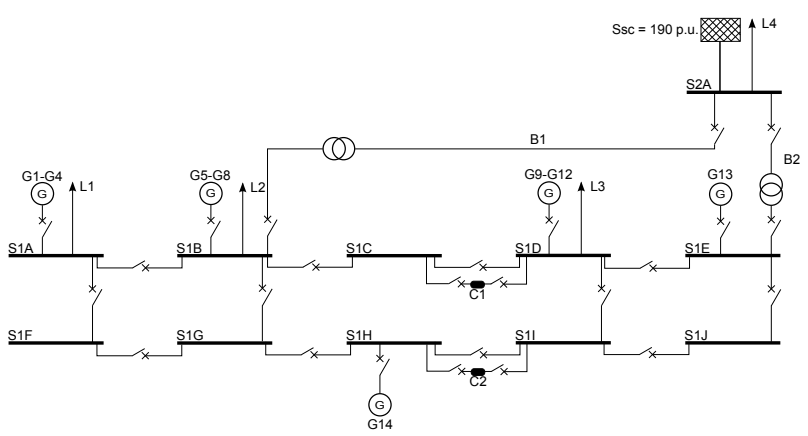

Figure 3. Test Case I: Large Industrial Substation

\section{B. Reliability and Non-Occurrence of Islanding}

In order to comply with the SCC, the algorithm is forced to open several circuit breakers. This could cause reliability and/or islanding issues. To mitigate this, the objective function (16) is such that the number of closed breakers is maximized. This results in a maximally interconnected system whilst still complying with the PFC and SCC.

$$
\text { maximize } \sum \delta
$$

Although this objective function assures that the optimal topology is as interconnected as possible, islanding could occur in this solution. To this end, a test is developed that determines whether islanding occurs. If islanding occurs, the solution is excluded from the solution space. Otherwise, it is considered as an optimal solution. The reason for not including the islanding test as a constraint is because the combination of the islanding equations and the binary variables result in highly complex nonlinear mixed integer constraints which would have a very detrimental effect on the calculation time. 


\section{Optimal Topology}

By applying the developed algorithm to this problem, the optimal topology depicted in Fig. 4 is found. The algorithm eliminated the need for the short circuit limiting reactors by opening two bus bar breakers, between S1C-S1D and S1HS1I. This solution satisfies the PFC and the SCC.

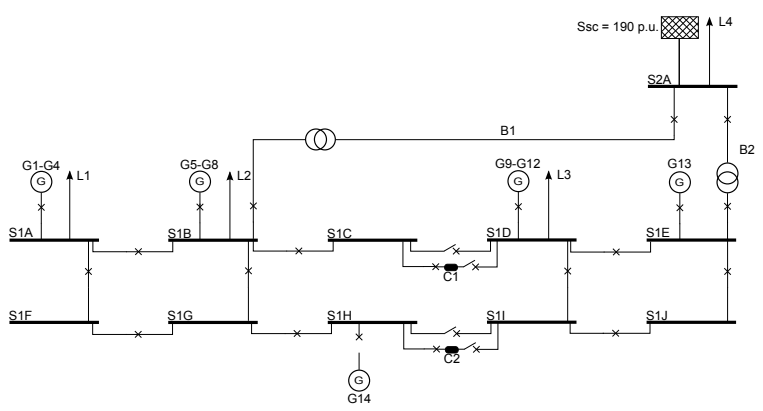

Figure 4. Solution for Test Case I

There is no islanding in the optimal topology nor are there $\mathrm{N}-1$ constraints violated by the proposed switching actions. It can be concluded that for this test case, bus bar switching as proposed in this paper can be a realistic and cheaper substitute for short circuit current limiting equipment

\section{Test CASE II: IEEE14 POWER SYSTEM}

In this section, the algorithm is applied to a power system with fourteen substations. The purpose of this test case is to show the effectiveness of the algorithm in finding an optimal topology for more complex power systems. As the size of the problem increases, it becomes increasingly difficult for a human operator to find a global solution where no other substations are affected by the actions taken in a certain substation. The computation time related to solving MINCQCPs increases dramatically when the size of the considered problem increases. To this end, a heuristic scheme is introduced in this section that decreases the computation time significantly.

\section{A. Power System based on IEEE14 Bus System}

The single node representation of the power system used in this test case is depicted in Fig. 5. This network consists of fourteen substations, five generators, eleven loads and twenty branches. The topology of this grid is based on the IEEE14 bus system. Each substation has two bus bars, A and B. All elements (e.g. generators, loads or branches) are connected to each bus bar by an individual circuit breaker with a corresponding $\delta^{A}$ and $\delta^{B}$.

In order to assure that an element e is only connected to one bus bar, an extra constraint (17) is introduced.

$$
\delta_{e}^{A}+\delta_{e}^{B}=1 \quad \forall \mathrm{e}
$$

As an example, the topology of substation 2 is shown in Fig. 6. The topology of the substations is simplified, only the circuit breakers relevant for this problem are considered.

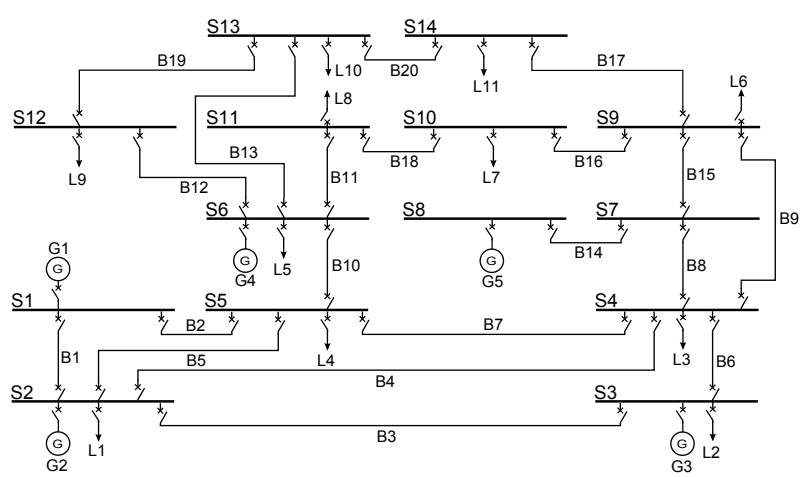

Figure 5. Test Case II: IEEE14 power system (single node representation)

\section{B. Heuristic Scheme}

A MINCQCP is in NP-complete [10], meaning that the computational time required to find an optimal solution for this problem increases at most exponentially as the size of the problem increases. One of the possibilities to address this is to introduce a heuristic strategy.

The heuristic strategy proposed in this paper initially limits the number of switchable circuit breakers to those of the substations with a too high short circuit power. This significantly reduces the number of mixed integer variables of the problem. If no solution can be found considering only those breakers, then the breakers of the substations connected to the substation with a too high short circuit power are considered as well. No constraints are eliminated from the problem by the heuristic scheme. Removal of constraints could result in the induction of short circuit violations in other substations due to switching actions in the considered substations. The choice to first consider switching actions in the substations with a too high short circuit power is based on the fact that those switching actions have the biggest impact on increasing the equivalent short circuit impedance and thus on lowering the short circuit power [13].

The flowchart of the heuristic scheme is depicted in Fig. 7. The first step of the heuristic scheme is to determine which substations experience a too high short circuit power. This is done by fixing the topology of the considered power system, reducing the problem to a linear program. This can easily be solved by CPLEX, resulting in the short circuit power levels for all substations.

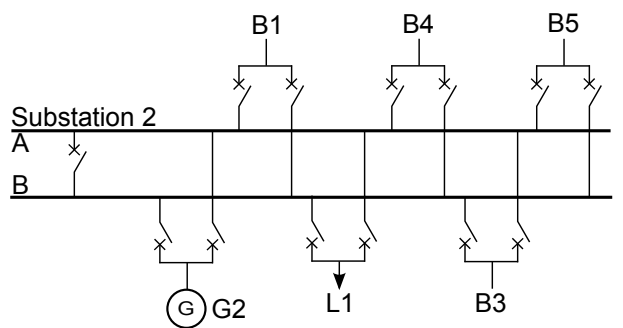

Figure 6. Test Case II: Substation 2 


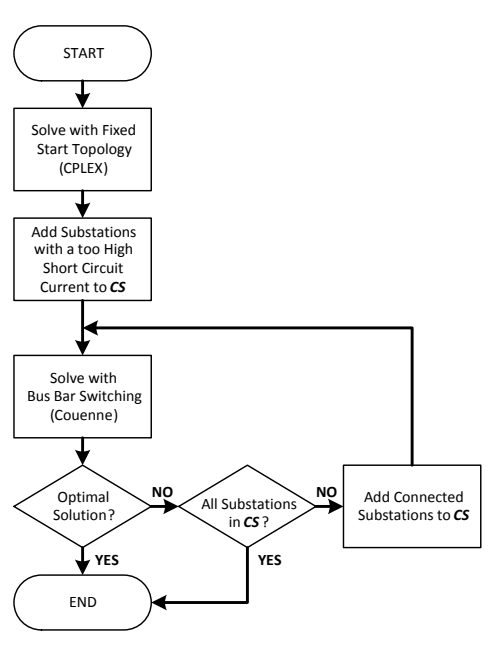

Figure 7. Heuristic Scheme

In the second step, all substations where the short circuit limitation of at least one circuit breaker is violated are added to the collection $\mathbf{C S}$.

In the next step, the topology of the substations in CS is adaptable and the topology of all other substations remains fixed. The reduced MINCQCP is then solved using Couenne. If no optimal solution is found, that satisfies both the constraints and the islanding test, then the substations connected to the substations in CS are added to CS. This loop stops if an optimal solution is found, if the user defined maximum number of loops is reached or if all substations are element of CS.

\section{Solution}

The first step of the heuristic scheme determines the short circuit levels of each substation. A short circuit level of 157 p.u. can be noticed on the bus bars of substation 2, which exceeds the limit of 152 p.u., leading to the addition of that substation to CS. No optimal solution can be found considering only bus bar switching actions in substation 2. All substations connected to substation 2 are then added to CS, these are substations 1, 3, 4 and 5. An optimal solution is found considering bus bar switching actions in those substations. The optimal topology of those five substation is depicted in Fig. 8. The topology of all other substations remains unchanged. The short circuit level of the bus bars A and B of substation 2 drop respectively to 132.4 and 26.3 p.u.. The total computation time of the heuristic scheme is 4 hours and 25 minutes.

Table II

Test CASE II: Computational time

\begin{tabular}{llll}
\hline Loop number & Calculation time & Number of $\delta^{\prime} \mathrm{s}$ & Optimal Solution \\
\hline 0 & $0.3 \mathrm{sec}$ & 0 & NO \\
1 & $4863.8 \mathrm{sec}$ & 13 & NO \\
2 & $11177.5 \mathrm{sec}$ & 53 & YES \\
\hline \hline TOTAL & $16041.6 \mathrm{sec}$ & & \\
\hline
\end{tabular}

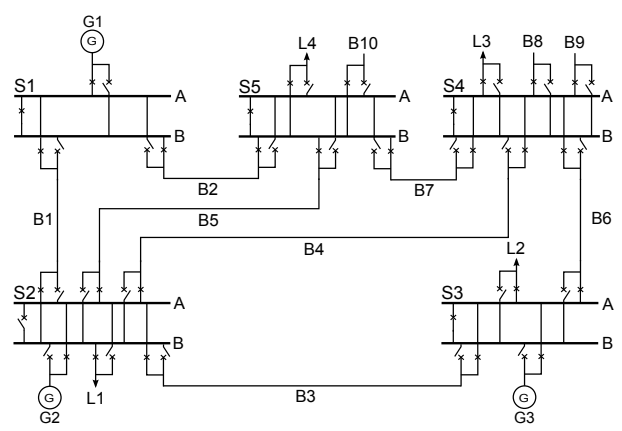

Figure 8. Test Case II: Solution

\section{CONCLUSION}

An optimization algorithm is presented in this paper which allows the reduction of the short circuit level using bus bar switching and hence contributes to the flexible operation of any power system. The optimization algorithm contains Power Flow Constraints (PFC) as well as Short Circuit Constraints (SCC). Two test cases show that the algorithm is able to adapt the topology of a grid in order to comply with both PFC and SCC. The type of problem is a MINCQCP, which is in NP-complete. A heuristic scheme is developed in order to reduce the long computation time linked to these type of problems. The heuristic scheme initially restricts the number of considered breakers that can be switched.

\section{REFERENCES}

[1] Y. Tada, H. Okamoto, A. Kurita, and Y. Sekine, "Analytical Methods for Determining a System Configuration Acceptable from Viewpoints of Both Short Circuit Current and Voltage Stability," Electrical Engineering in Japan, Vol. 124, No. 3, pp. 30-39, 1998.

[2] H. G. Sarmiento, R. Catellanos, C. Tovar, and G. Pampin, "An Example in Controlling Short Circuit Levels in a Large Metropolitan Area," IEEE Publications 0-7830-7989-6/03, pp. 589-594, Jul. 2003.

[3] H. Seyedi, and B. Tabei, "Appropriate Placement of Fault Current Limiting Reactors in Different HV Substation Arrangements," Circuits and Systems, pp. 252-262, Jul. 2012.

[4] A. A. Mazi, B. F. Wollenberg, and M. H. Hesse, "Corrective Control of Power System Flows by Line and Bus Bar Switching," IEEE Transactions on Power Systems, Vol. 1, pp. 258-264, Aug. 1986.

[5] H. J. Koglin, and H. Muller, "Corrective Switching: a New Dimension in Optimal Load Flow," International Journal of Electrical Power and Energy Systems, Vol. 4, pp. 142-149, Apr. 1982.

[6] W. Shao, and V. Vittal, "Corrective Switching Algorithm for Relieving Overloads and Voltage Violations," IEEE Transactions on Power Systems, Vol. 20, No. 4, pp. 1877-1885, Nov. 2005.

[7] L. Chen, H. Tozyo, Y. Tada, H. Okamoto, and R. Tanabe, "Reconfiguration of Transmission Systems with Transient Stability Constraints," IEEE Publications 0-7830-5935-6/00, pp. 1320-1324, 2000.

[8] E. B. Fisher, R. P. O'Neill, and M. C. Ferris, "Optimal Transmission Switching," IEEE Transactions on Power Systems, Vol. 23, No. 3, pp. 1346-1355, Aug. 2008.

[9] T. Van Acker, "Implementation of Bus Bar Switching in Optimal Power Flow Problems," Master Thesis, Dept. ESAT (ELECTA), KU Leuven, Belgium, 2014

[10] A. D. Pia, S. S. Dey, and M. Molinaro, "Mixed-integer Quadratic Programming is in NP," Cornell University Library, Jul. 2014.

[11] R. Fourer, D. M. Gay, and B. W. Kernighan, AMPL: A Modeling Language for Mathematical Programming, Duxbury, 2003, p.xv

[12] P. Belotti, "COUENNE: a user's manual," Dept. of Mathematical Sciences, Clemson University, n.d.

[13] J. Grainger, and W. D. Stevenson Jr., Power System Analysis, Mc Graw Hill, 1994, p. 395. 